\title{
Orally Disintegrating Film Dosage Form
}

National Cancer Institute

\section{Source}

National Cancer Institute. Orally Disintegrating Film Dosage Form. NCI Thesaurus. Code C149729.

Solid preparation consisting of a single- or multilayer sheet of suitable material(s) intended to be placed in the mouth where it disperses rapidly before being swallowed. 